\section{Reducing wing observability to radar using microserrations at the leading edge}

\author{
Karri Sai Krishna Reddy and Avijit Chatterjee* \\ Department of Aerospace Engineering, Indian Institute of \\ Technology Bombay, Powai, Mumbai 400 076, India
}

Serrations are found in nature on the leading edge (LE) of bird wings and whale flippers. These serrations reduce flight noise and improve aerodynamic performance. This has inspired LE serrations to be incorporated in the design of aircraft wings and turbine blades. LE of wings can contribute significantly to observability of aircraft by radar due to LE diffraction of horizontally polarized incident electromagnetic (EM) waves. Serrations at the wing LE can also be used to reduce observability to radar under such circumstances. In the present study, the method of equivalent currents is used to analyse the effect of microserrations on an infinitesimally thin metallic delta wing. It is shown that microserrations at the wing $L E$ can be effectively used to control backscatter due to diffraction of EM waves at the wing LE. LE serrations, in general, work by introducing a randomness in the interaction of wing $L E$ with incident fluid flow or EM field.

Keywords: Computational electromagnetics, leading edge, radar cross-section, serrations, wing observability.

SERRATIONS on the leading edge (LE) of bind wings and whale flippers serve as flow-control devices in nature. LE serrations reduce flight noise and enhance aerodynamic (or hydrodynamic) performance. LE comb-like serrations present in the barn owl wings are one of the three major adaptations responsible for its silent flight ${ }^{1}$. LE adaptation in the form of tubercles on flippers of humpback whales can delay onset of stall leading to superior performance in turn manoeuvres ${ }^{2}$. There is an increasing tendency to mimic LE serrations observed in nature ${ }^{3}$. The role of wing LE serrations is mostly to generate streamwise vorticity in the incident flow. This has multiple consequences, including energizing the boundary layer and converting periodic or near-periodic wake at LE into random behaviour. The latter is thought to be responsible for eliminating tonal noise in the wings with LE serrations compared to without, when a wing interacts with oncoming gust ${ }^{4}$.

The LE of a wing produces a noticeable glint in terms of visibility to radar, when it interacts with horizontally polarized electromagnetic (EM) field incident from or near nose-on direction. The glint is a spike in the value of radar cross-section (RCS), the quantitative measure of

*For correspondence. (e-mail: avijit@aero.iitb.ac.in) observability to radar, due to edge diffraction at LE involving horizontally polarized incident EM field. This is attributed to the presence of a major lobe in the Keller cone direction due to edge diffraction of incident horizontally polarized EM wave at the wing $\mathrm{LE}^{5}$. This glint is disadvantageous for contemporary combat aircraft designed with low observability to radar as a key design feature. In the present study we have used small or microserrations at the LE of a common combat aircraft wing geometry to reduce the glint caused due to interaction with horizontally polarized EM field. As in the case of elimination of tonal noise in the interaction of a gust with wing geometry, the idea is to introduce an element of randomness in the interaction of LE with the incident field due to the presence of small serrations at the LE. Serrations are used to break up the continuous wing LE to reduce lobe intensity, which is usually a function of the length of the straight, uninterrupted $\mathrm{LE}^{5}$. In the interaction of EM waves with aircraft type-configuration, backscatter contributions from edge-type discontinuities are usually higher-order effects compared to pure specular returns, but can become important at the wing LE and the trailing edge (TE) for specific orientation and polarization of the incident field. A similar study was conducted earlier to show the effectiveness of TE serrations in reducing the relatively prominent backscatter caused due to surface travelling waves interacting with edge discontinuity at the wing TE for vertically polarized EM field ${ }^{6}$.

In the present study a thin metallic delta wing with LE sweep of $45^{\circ}$ (also utilized in other studies ${ }^{6,7}$ ) was initially used to demonstrate the enhanced RCS due to horizontally polarized EM waves compared to vertical polarization for backscatter involving LE diffraction. This was done using both frequency domain-based method of moments $(\mathrm{MoM})^{5}$ and time domain-based finite volume time domain (FVTD) ${ }^{8}$ method. Both MoM and FVTD are fullwave solvers for numerical solution of the Maxwell's equations. The delta wing with added microserrations and zero thickness was then analysed using the method of equivalent currents (MEC) $)^{5,9}$. In high-frequency regimes,

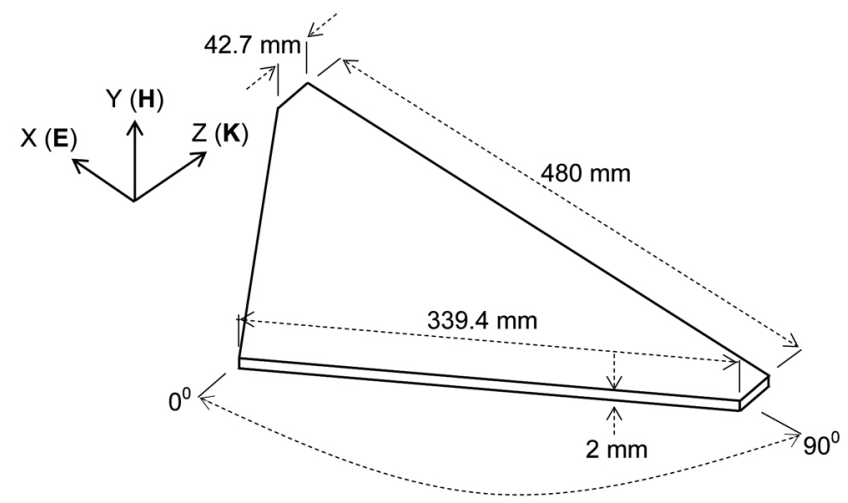

Figure 1. Plain delta wing used in the study. 

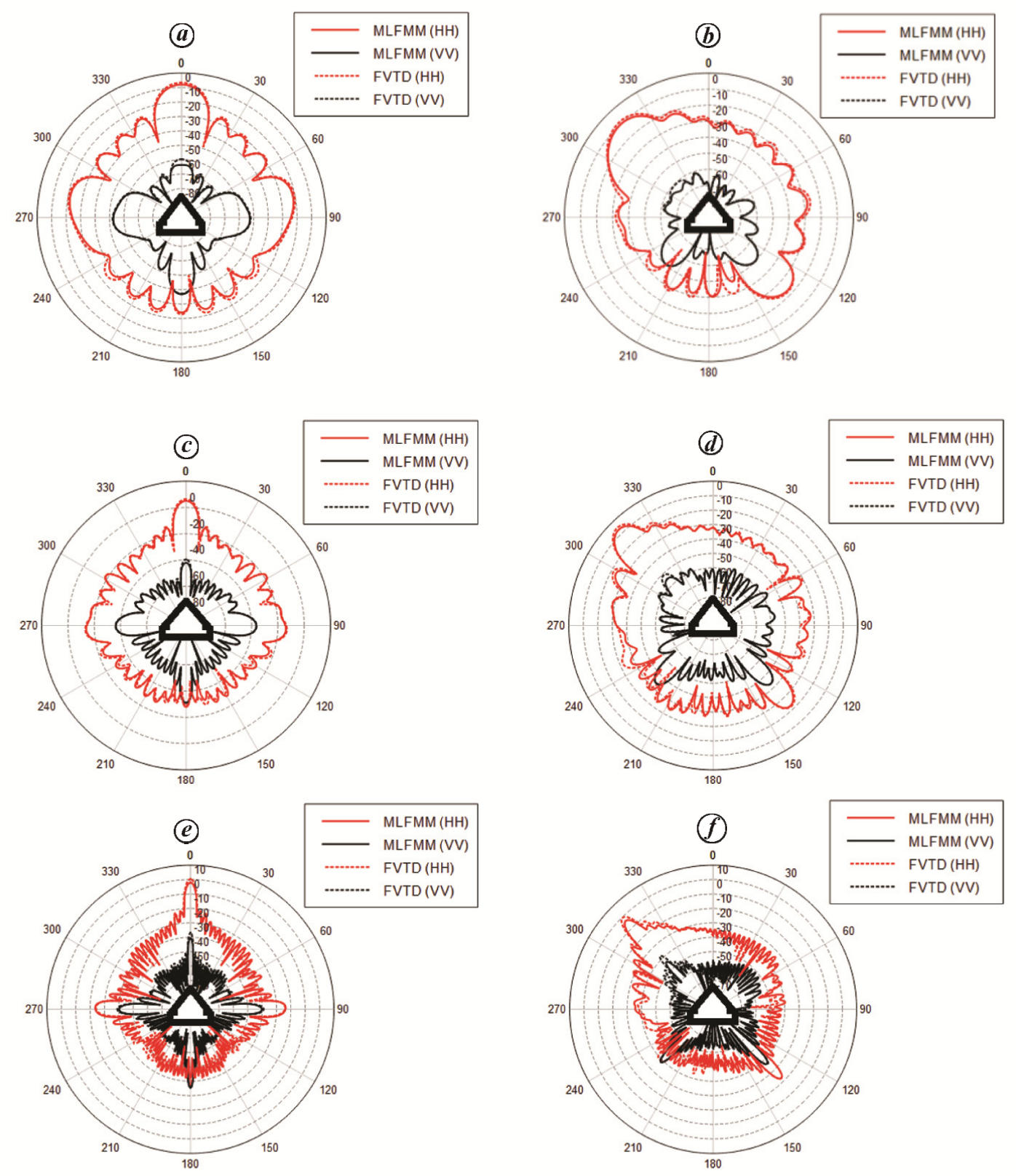

Figure 2. Bistatic polar plots, yaw plane, vertically polarized (VV) and horizontally polarized (HH), electromagnetic (EM) waves: $\boldsymbol{a}, 0^{\circ}$ incidence, frequency $2.5 \mathrm{GHz} ; \boldsymbol{b},-45^{\circ}, 2.5 \mathrm{GHz} ; \boldsymbol{c}, 0^{\circ}, 5 \mathrm{GHz} ; \boldsymbol{d},-45^{\circ}, 5 \mathrm{GHz} ; \boldsymbol{e}, 0^{\circ}, 10 \mathrm{GHz} ; \boldsymbol{f},-45^{\circ}$, $10 \mathrm{GHz}$.

MEC allows us to isolate and identify the contribution of LE diffraction by considering LE to be infinitesimally thin. Full-wave solvers like MoM and FVTD are not convenient and can be inaccurate or even fail in the presence of microserrations because of the very fine discretization required to resolve the geometry ${ }^{10}$. This is in addition to both full-wave methods being computationally expensive at high frequencies and fine discretizations. Delta wings usually have thin LE to promote flow separation for generating additional vortex lift due to stable vortex systems on the upper surface of the wing. Thus, infinitesimally thin LE for calculation of RCS using MEC is a reasonable assumption for the delta wing considered here. For a thin but finite thickness LE, as in Figure 1, RCS analysis would require additional physical optics (PO)-based formulations to take care of the specular contribution with edge diffraction effects computed using MEC as before.

Figure 1 shows the metallic delta wing without serrations. The validation of MoM in multi-level fast multipole method (MLFMM) form as well as the FVTD method is carried out with available results in the 
literature $^{6,7}$ at a frequency of $5 \mathrm{GHz}$. This frequency corresponds to wing span $b=8 \lambda$, where $\lambda$ is the wavelength of the incident harmonic EM wave. The validation results are presented in Teja et $a l .{ }^{6}$, and are not repeated here. It is well known that the LE of a wing has a large contribution to the overall RCS for horizontal polarization for $b / \lambda \gg 1$, due to diffraction at the wing $\mathrm{LE}^{5}$. Polar plots in Figure $2 a-f$ compare the bistatic RCS at 2.5, 5 and $10 \mathrm{GHz}$, in the plane of the wing, for vertical and horizontal polarization for nose-on incidence $\left(0^{\circ}\right)$ and broadside incidence to the $\operatorname{LE}\left(-45^{\circ}\right.$ or $\left.315^{\circ}\right)$. In all cases the horizontally polarized incident field results in a much larger RCS contribution, especially at the monostatic point. This is uniformly true, irrespective of the type of full-wave solver used for the simulation.

To isolate LE diffraction contributions to the overall RCS, the delta wing is made infinitesimally thin and LE contribution evaluated using MEC both for serrated and unserrated LE. Figure 3 shows the delta wing geometry with microserrations at the LE. The contribution to RCS due to edge diffraction of horizontally polarized EM waves at a straight LE is proportional to $L^{2}$, where $L$ is

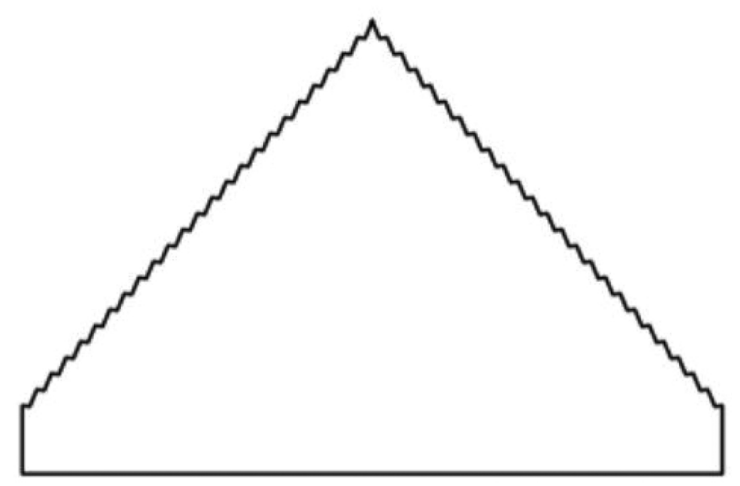

Figure 3. Delta wing with leading edge (LE) serrations.

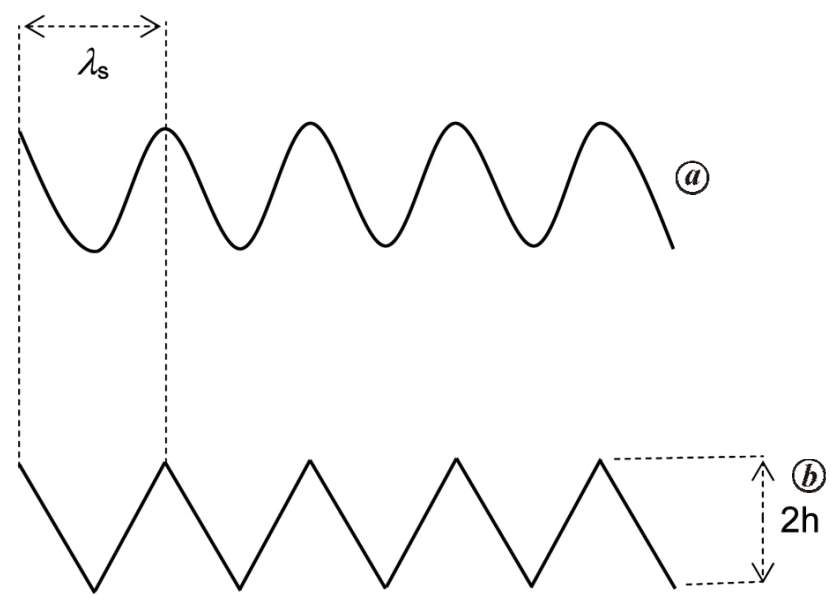

Figure 4. Geometry of serrations: (a) cosine curve and (b) cosine curve approximated by straight lines for actual geometry. the length of the $\mathrm{LE}^{5}$. Serrations in Figure 3, in principle, seek to break length $L$ into smaller line segments to reduce backscatter. The LE serrations conceptually in the form of a cosine curve of wavelength $\lambda_{\mathrm{s}}$ and height $2 h$ (Figure $4 a$ ) are approximated for the present study as triangular serrations (Figure $4 b$ ). The height $2 h$ is expressed as a small fraction of the wing root chord $C$, while $\lambda_{\mathrm{s}}$ is a fraction of the wavelength of the incident harmonic wave $\lambda$. Full-wave solvers will have difficulty in resolving LE serrations for $2 h \ll C$. The computational time would also be prohibitively large for such solvers, given the very fine discretization required to adequately resolve the serrations. MEC, on the other hand, assumes edges to be formed by the intersection of two semiinfinite surfaces. The surface current generated on the edges, also known as equivalent currents, are calculated based on diffraction coefficients, relative position of the edge with respect to the observer and the incident electric field $^{5,9}$. Once equivalent currents are calculated for individual serrations, they can be summed up in the standard far-field integral ${ }^{11}$ and the RCS calculated. Figure 5 shows the validation of MEC method for a metallic equilateral triangle with sides approximately $9 \lambda$ and horizontal polarization with results in literature ${ }^{12}$.

Figure $6 a-c$ shows results in terms of monostatic plots obtained using the MEC method for serrated and unserrated, infinitesimally thin delta wing for 12.5, 15 and $20 \mathrm{GHz}$ respectively. At these frequencies the wing span measures $20 \lambda, 24 \lambda$ and $32 \lambda$ respectively. The serration depth is taken to be only $2 \%$ of the maximum wing chord to cause minimum disruption by the introduced LE serrations to the incident fluid flow. In this range of frequency and fixed serration depth, a serration wavelength $\lambda_{\mathrm{s}}=0.5 \lambda$ shows the best performance with reduction of around $10 \mathrm{dBsm}$ at broadside $\left( \pm 45^{\circ}\right)$ incidence (Figure $6 a-c)$. This reduction in RCS can be attributed to the breaking up of LE and consequently of the major lobe formed by edge diffraction due to uninterrupted LE. In

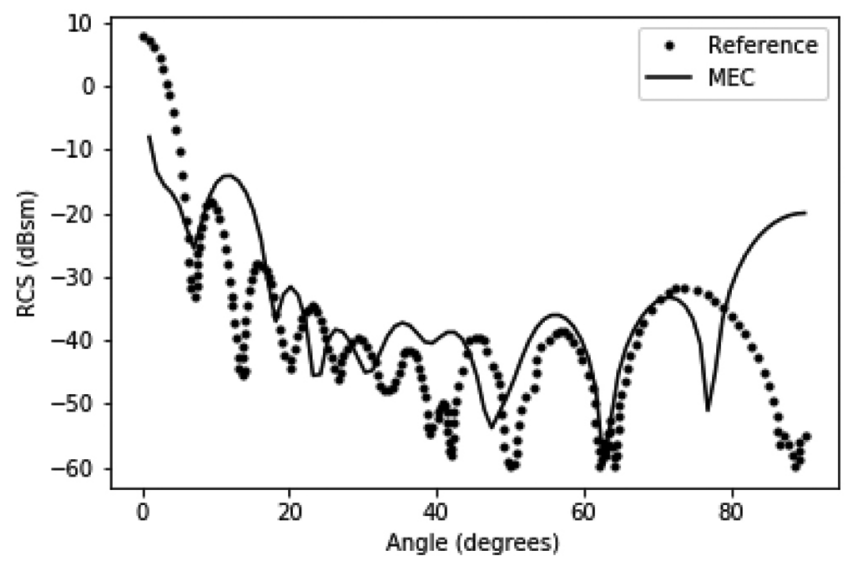

Figure 5. Validation of method of equivalent currents results, equilateral triangular plate. 


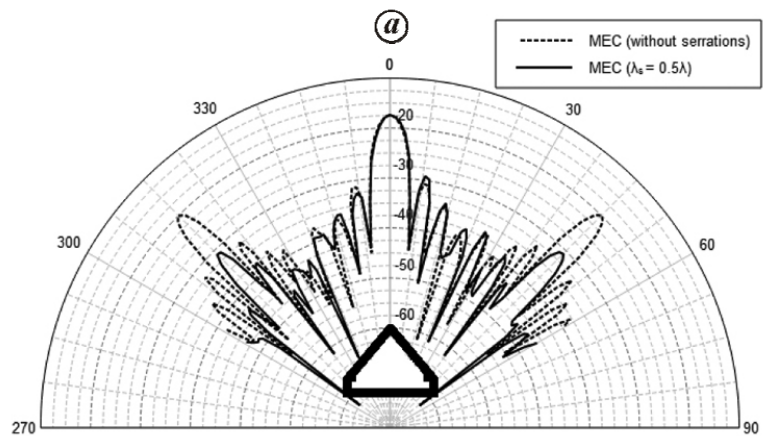

$\operatorname{RCS}(\mathrm{dBsm})$

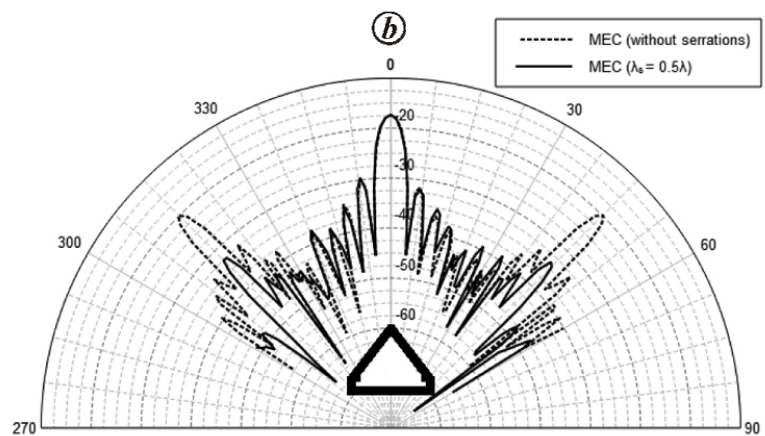

$\operatorname{RCS}(\mathrm{dBsm})$

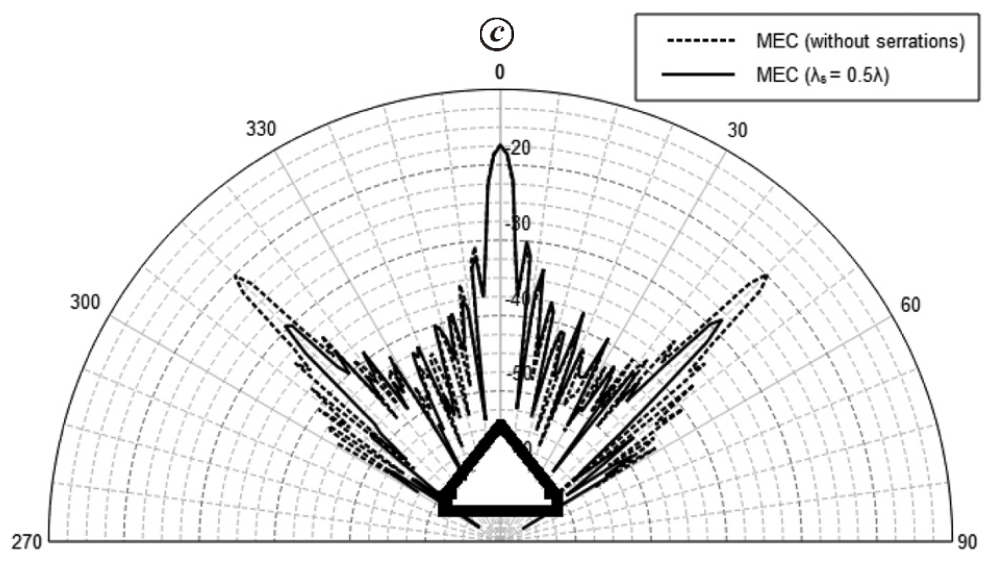

$\operatorname{RCS}(\mathrm{dBsm})$

Figure 6. Monostatic radar cross-section, LE serrated wing, HH polarization: (a) 12.5, (b) 15 and (c) $20 \mathrm{GHz}$.

addition, the serrated edge also results in multiple nonaligned scattering centres, which scatter energy away from the monostatic point. However, for best performance the serration depth and wavelength of LE serrations need to be optimized rigorously for the operating range of frequencies.

1. Lilley, G. M., A study of the silent flight of the owl. In 4th AIAA/CEAS Aeroacoustics Conference, Toulouse, France, AIAA Paper 1998-2340, 1998.

2. Fish, F. E., Weber, P. W., Murray, M. M. and Howle, L. E., The tubercles on humpback whales' flippers: application of bioinspired technology. Integr. Comp. Biol., 2011, 51, 203-213.

3. Choi, H., Park, H., Sagong, W. and Lee, S.-I., Biomimetic flow control based on morphological features of living creatures. Phys. Fluids, 2012, 24, 121302-1-121302-20.

4. Hersh, A. S., Soderman, P. T. and Hayden, R. E., Investigation of acoustic effects of leading-edge serrations on airfoils. J. Aircraft, 1974, 11, 197-202.

5. Knott, E. F., Shaeffer, J. and Tuley, M., Radar Cross Section. Institution of Engineering and Technology, Electromagnetics and Radar Series, SciTech Publishing, 2004, 2nd edn, pp. 200-267.

6. Teja, T. S., Vaghela, M. B. and Chatterjee, A., Reducing aircraft radar cross-section with owl wing type serrated trailing edge. Curr. Sci., 2017, 112, 1020-1023.

7. Lu, L.-J., Chen, H.-Y., Zhou, P.-H., Liang, D.-F. and Deng, L.-J., Design of controlling edge scattering based on tapered periodic surfaces loading. In Proceedings of Progress in Electromagnetics Research Symposium, Guangzhou, China, 2014.

8. Rao, S. M., Time Domain Electromagnetics, Academic Press, San Diego, 1999, pp. 307-365.

9. Michaeli, A., Equivalent edge currents for arbitrary aspects of observation. IEEE Trans. Antennas Propag., 1984, 32, 252-258.

10. Panayappan, K. and Mittra, R., A technique for handling multiscale electromagnetic problems using the finite difference time domain (FDTD) algorithm. J. Electromag. Waves Appl., 2016, 30, 1241-1264.

11. Balanis, C. A., Advanced Engineering Electromagnetics, John Wiley, New York, 1989, pp. 285-291.

12. Van Ewijk, L. J., Diffraction computation by means of the method of equivalent currents. TNO Physics and Electronics Laboratory report, The Hague, Netherlands, FEL-94-B195, 1994.

Received 20 August 2018; revised accepted 21 February 2019

doi: $10.18520 / \mathrm{cs} / \mathrm{v} 116 / \mathrm{i} 10 / 1731-1734$ 